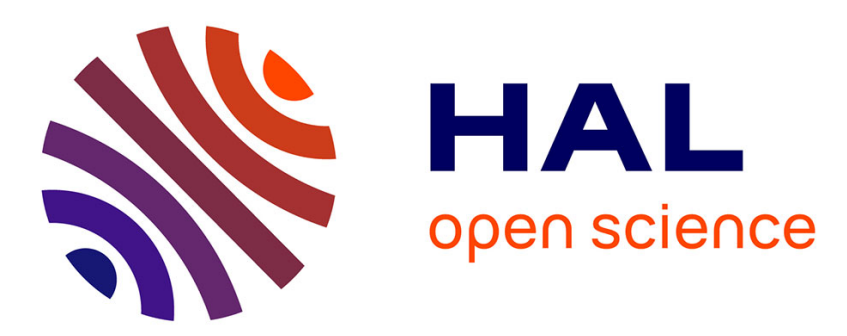

\title{
Modification d'un ensemble de source et d'injection a ions négatifs pour tandem
}

\author{
B. Delaunay, J.L. Girma
}

\section{To cite this version:}

B. Delaunay, J.L. Girma. Modification d'un ensemble de source et d'injection a ions négatifs pour tandem. Revue de Physique Appliquée, 1969, 4 (2), pp.171-172. 10.1051/rphysap:0196900402017100 . jpa-00243201

\section{HAL Id: jpa-00243201 https://hal.science/jpa-00243201}

Submitted on 1 Jan 1969

HAL is a multi-disciplinary open access archive for the deposit and dissemination of scientific research documents, whether they are published or not. The documents may come from teaching and research institutions in France or abroad, or from public or private research centers.
L'archive ouverte pluridisciplinaire HAL, est destinée au dépôt et à la diffusion de documents scientifiques de niveau recherche, publiés ou non, émanant des établissements d'enseignement et de recherche français ou étrangers, des laboratoires publics ou privés. 


\title{
MODIFICATION D'UN ENSEMBLE DE SOURGE ET D'INJEGTION A IONS NÉGATIFS POUR TANDEM
}

\author{
B. DELAUNAY et J. L. GIRMA, \\ Service de Physique Nucléaire à Basse Énergie, G.E.N., Saclay.
}

\begin{abstract}
Résumé. - Plusieurs modifications ont été faites au tandem de Saclay pour obtenir des ions ${ }^{3} \mathrm{He}$. Le courant analysé est de $230 \mathrm{nA}$ d'ions ${ }^{3} \mathrm{He}$ de $18 \mathrm{MeV}$ avec échange dans l'hydrogène.
\end{abstract}

Abstract. - Several modifications have been made to at the tandem to obtain ${ }^{3} \mathrm{He}$ beams. The analyzed current is $230 \mathrm{nA}, 18 \mathrm{MeV}{ }^{3} \mathrm{He}$ with charge-exchange in hydrogen.

I. Conditions de fonctionnement. - Le tandem $12 \mathrm{MeV}$ HVEG a été installé à Saclay en 1963 . Il peut accélérer normalement des protons et des deutons. Pour les autres particules, il est nécessaire de rechercher la meilleure solution et principalement pour la source d'ions négatifs et l'injection. Pour obtenir des faisceaux d'hélium-3, il existe plusieurs méthodes résumées dans

\section{TABLEAU I}

Différentes méthodes pour obtenir des ${ }^{3} \mathrm{He}^{-}$

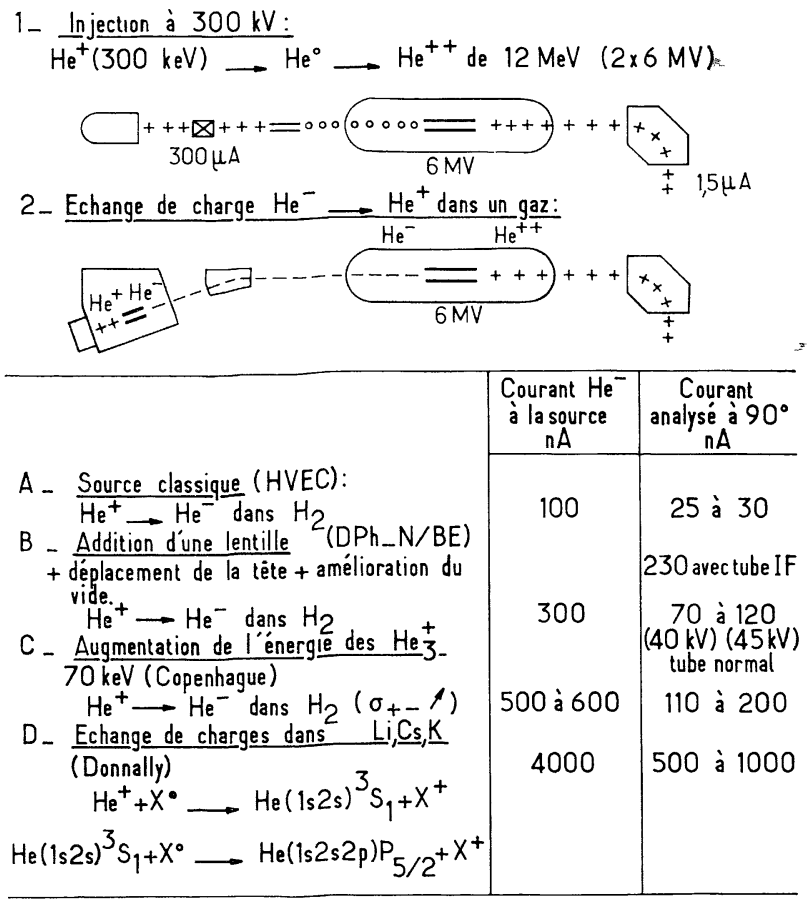

le tableau I. Nous avons d'abord choisi de rechercher les conditions les meilleures en conservant l'échange à l'hydrogène et la source standard HVEC. Nous avons obtenu $230 \mathrm{nA}$ d'hélium-3 analysés (dans 2,5 mm) à $18 \mathrm{MeV}$ dans les conditions ci-après :

1) Source d'ions : a) un système simple à vis permet la translation de la « tête » de source afin de rechercher la position optimale; $b$ ) la chambre à arc en molybdène permet une plus longue durée; c) les pièces de l'ensemble d'extraction sont interchangeables; d) des refroidissements supplémentaires assurent une meilleure stabilité de l'ensemble.

2) Une lentille additionnelle « uni-électrode » a été installée en vue d'améliorer la focalisation avant l'analyse à $20^{\circ}$.

3) Une nouvelle chambre d'analyse à $20^{\circ}$ permet d'abaisser la pression dans la zone de l'analyse des ions hélium de $80 \mathrm{keV}$.

4) Une nouvelle lentille de $5^{\prime \prime}$ à grilles remplace celle de $2^{\prime \prime}$ afin de diminuer les aberrations et assurer une meilleure transmission.

5) Le système de vide a été remplacé en basse et haute énergie par une pompe turbo-moléculaire de $500 \mathrm{l} / \mathrm{s}$ et une pompe à mercure de $800 \mathrm{l} / \mathrm{s}$ utilisables ensemble ou séparément. Le vide est amélioré en basse énergie; à haute énergie, le flux de gaz d'échange est augmenté.

6) Le tube accélérateur normal a été remplacé par un tube à " champ incliné ". La transmission est passée de $16 \%$ à $25 \%$ à $18 \mathrm{MeV}$; au total, le faisceau qui était de l'ordre de 20 à $30 \mathrm{nA}$ dans les conditions initiales est passé à environ $150 \mathrm{nA}$ pour $1,25 \mathrm{~mm}$ et $230 \mathrm{nA}$ pour $2,5 \mathrm{~mm}$.

II. Récupération de l'hélium-3. - Un appareillage a été construit pour recueillir le mélange ${ }^{3} \mathrm{He}+\mathrm{H}_{2}$, séparer les impuretés et récupérer ${ }^{3} \mathrm{He}$ par recombi- 
naison de $\mathrm{H}_{2}$ sur de l'oxyde de cuivre [1]. Pour un mélange de $39,8 \mathrm{l}$ (1 atm.), on a récupéré $7,1 \mathrm{l}$ de ${ }^{3} \mathrm{He}$ à $99 \%$.

III. Nouvelle source. - Une nouvelle source a été construite avec les caractéristiques suivantes : a) les boules isolantes ont été remplacées par des colonnes isolantes [2]. Les essais en protons ont montré les mêmes performances; $b$ ) le «nez »d'extraction a été rendu déplaçable longitudinalement, ce qui permettra d'augmenter et d'ajuster la tension d'extraction; c) un four à lithium a été construit. Les essais sur banc montrent une bonne condensation dans les pièges et une bonne tenue thermique et électrique.

Note sur épreuve. - État au 18 juin 1969 : cette source est en fonctionnement au centre de Bruyèresle-Châtel.

\section{BIBLIOGRAPHIE}

[1] Deiraunay (B.) et Gaudron (C.) (à paraître).

[2] Rapport de l'Université de Pennsylvanie (U.S.A.). 